A Generalized 2D-Dynamical Mean-Field Ising Model with a Rich Set of Bifurcations (Inspired and Applied to Financial Crises)

Journal Article

Author(s):

Smug, Damian; Sornette, Didier; Ashwin, Peter

Publication date:

2018-04

Permanent link:

https://doi.org/10.3929/ethz-b-000265138

Rights / license:

Creative Commons Attribution 4.0 International

Originally published in:

International Journal of Bifurcation and Chaos 28(4), https://doi.org/10.1142/S0218127418300100 
International Journal of Bifurcation and Chaos, Vol. 28, No. 4 (2018) 1830010 (14 pages)

(C) The Author(s)

DOI: 10.1142/S0218127418300100

\title{
A Generalized 2D-Dynamical Mean-Field Ising Model with a Rich Set of Bifurcations (Inspired and Applied to Financial Crises)
}

\author{
Damian Smug* \\ Centre for Systems, Dynamics and Control, \\ Department of Mathematics, Harrison Building, \\ University of Exeter, Exeter EX4 4QF, UK \\ d.smug@exeter.ac.uk \\ Didier Sornette \\ ETH Zurich, Department of Management, \\ Technology and Economics, Scheuchzerstrasse 7, \\ CH-8092 Zürich, Switzerland \\ Swiss Finance Institute, c/o University of Geneva, \\ 40 blvd. Du Pont d'Arve, CH 1211 Geneva 4, Switzerland \\ dsornette@ethz.ch \\ Peter Ashwin \\ Centre for Systems, Dynamics and Control, \\ Department of Mathematics, Harrison Building, \\ University of Exeter, Exeter EX4 4QF, UK \\ p.ashwin@exeter.ac.uk
}

Received February 8, 2018

\begin{abstract}
We analyze an extended version of the dynamical mean-field Ising model. Instead of classical physical representation of spins and external magnetic field, the model describes traders' opinion dynamics. The external field is endogenized to represent a smoothed moving average of the past state variable. This model captures in a simple set-up the interplay between instantaneous social imitation and past trends in social coordinations. We show the existence of a rich set of bifurcations as a function of the two parameters quantifying the relative importance of instantaneous versus past social opinions on the formation of the next value of the state variable. Moreover, we present a thorough analysis of chaotic behavior, which is exhibited in certain parameter regimes. Finally, we examine several transitions through bifurcation curves and study how they could be understood as specific market scenarios. We find that the amplitude of the corrections needed to recover from a crisis and to push the system back to "normal" is often significantly larger than the strength of the causes that led to the crisis itself.
\end{abstract}

Keywords: Ising model; social opinion dynamics; chaos; regime shifts; bifurcation delay.

\footnotetext{
*Author for correspondence

This is an Open Access article published by World Scientific Publishing Company. It is distributed under the terms of the Creative Commons Attribution 4.0 (CC-BY) License. Further distribution of this work is permitted, provided the original work is properly cited.
} 


\section{Introduction}

The Ising model and its mean-field version have a time-honored history in economics, sociology and finance, since its introduction as a mathematical model of ferromagnetism in statistical mechanics in 1920. Rather than magnetic spins related via Heisenberg interactions, the spins represent agents who have several options and decide to adopt one of them according to a combination of inputs involving their own idiosyncratic judgments (akin to thermal noise in physics), external news (similar to the external magnetic field) and social influences (analogous to the spin-spin exchange interaction). A large set of economic models can be mapped onto various versions of the Ising model to account for social influence in individual decisions. And the Ising model is one of the simplest models describing the competition between the ordering force of imitation or contagion and the disordering impact of private information or idiosyncratic noise. It is sufficiently rich to exhibit complex behaviors, such as phase transitions (bifurcations) and spontaneous symmetry breaking [McCoy \& Wu, 1973]. Since decision making and social interactions are two of the most important ingredients of social organization, it is thus natural that the Ising model and its extensions to understand social organization have blossomed over many decades (see e.g. Brock \& Durlauf, 2001; Callen \& Shapero, 1974; Galam et al., 1982; Galam \& Moscovici, 1991; Gordon et al., 2009; Granovetter \& Soong, 1983; Montroll \& Badger, 1974; Nadal et al., 2005; Orléan, 1995; Phan et al., 2004; Schelling, 1971; Sornette, 2014: Weidlich. 1971. 1991. 2003: Weidlich \& Huebner, 2008]).

Motivated by its applications to financial markets, we study an extended version of the dynamical mean-field equation of the Ising model in which the external (magnetic or news) field is endogenized to represent a smoothed moving average of the past state variable. This new model stands for a simplification of the interplay between instantaneous social imitation and past trends in social coordinations Harras et al., 2012: Kaizoii et al. 2015: Sornette \& Zhou, 2006; Zhou \& Sornette, 2007]. We show the existence of a rich set of bifurcations as a function of the two parameters quantifying the relative importance of immediate versus past social opinions on the formation of the next value of the state variable. Moreover, we identify where one can find chaos in the 3D parameter space. Finally, we explore how the parameter shifts through certain bifurcation curves lead to variations in the behavior of the system.

The article is organized as follows. The next section recalls the equation and main properties of the standard mean-field Ising model. Section 3 introduces the extended mean-field Ising model, which takes the form of two coupled discrete equations. In Sec. 4. we analyze bifurcations in the extended system and, in Sec. 5, we compare the behavior to the original 1D system. Section 6 presents where one can find chaotic behavior in the extended system. Section 7 covers certain scenarios of market passages when parameters changed from one regime to another. Section 8 concludes.

\section{Dynamical Version of the Standard Mean-Field Ising Model}

The standard mean-field equation of the Ising model can be written as

$$
s=\tanh [\beta(s+H)],
$$

where $s$ is the average state variable (mean spin or magnetization) of a given representative agent and $H$ is the external influence (magnetic field). In financial applications, $s$ can represent the traders' opinions whether to buy or sell and $H$ is the impact of incoming news on their decisions. Parameter $\beta$ quantifies the strength of the social imitation between agents (or, originally, the inverse temperature). Expression (1) describes the value of the average opinion $s$ as being determined by the sum of the external influence $H$ and of an effective impact exerted by the other surrounding agents, themselves adopting on average the same opinion $s$. The average state variable $s$ is thus the solution of the implicit equation (1), which has two control parameters $\beta$ and $H$.

In a dynamical context, one can generalize (10) into a recurrence equation describing how the collective opinion evolves, when influenced by external news and its past state Ollikainen, 2016]:

$$
s_{t+1}=\tanh \left[\beta\left(s_{t}+H\right)\right] .
$$

Then, the fixed points of the map (21) are solutions of the implicit equation (1). Figure 1 presents how the fixed points of the map (2) vary when the parameters $H$ and $\beta$ change. In the symmetric case for $H=0$ [Figs. 1(a) and 1(b)], there is a pitchfork bifurcation at $\beta=1$, hence, when the system passes 


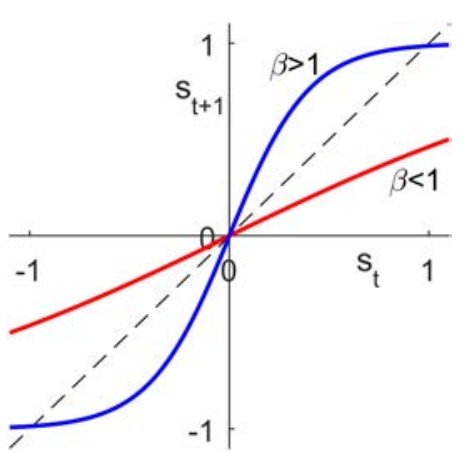

(a)

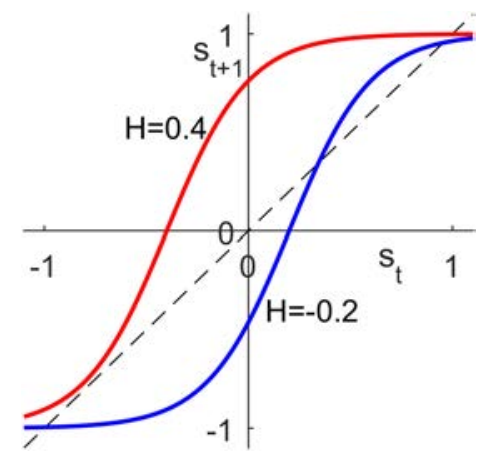

(c)

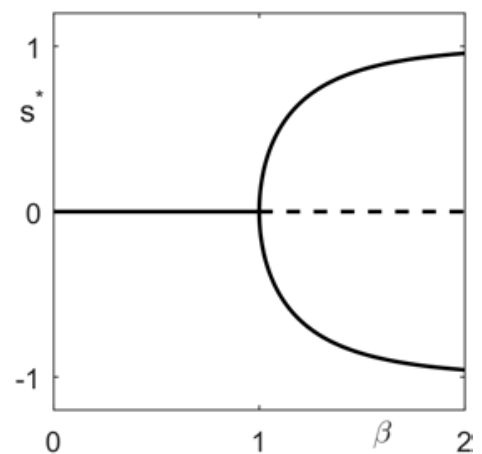

(b)

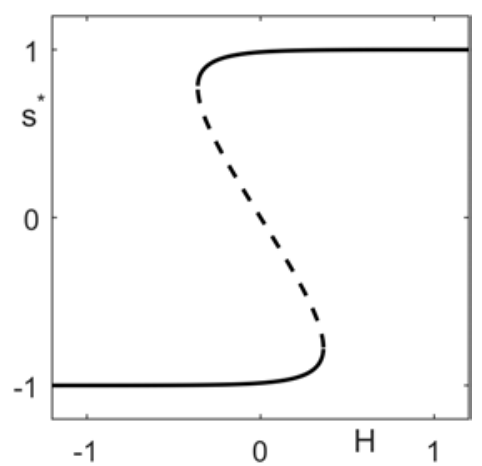

(d)

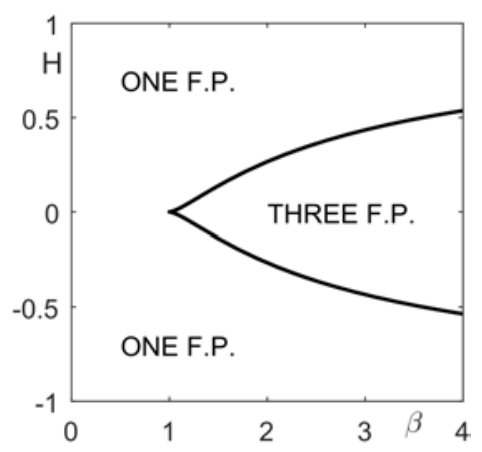

(e)

Fig. 1. The map (2) $s_{t+1}=\tanh \left[\beta\left(s_{t}+H\right)\right]$ and its bifurcation diagrams for different values of the coupling strength $\beta$ and external field $H$. (a) $H=0$. Depending on $\beta$ the shape of the map changes. Higher values of $\beta$ increase the curvature of the map giving birth to two new fixed points at $\beta=1$. The schematic curves are plotted for $\beta=0.5$ (red) and $\beta=2.5$ (blue). (b) Bifurcation diagram for $H=0$ with a pitchfork bifurcation at $\beta=1$. (c) $\beta=2.5$. Depending on $H$ the function is moved horizontally. For $\beta>1$ such a shift can make some of the fixed points disappear in a saddle-node bifurcation. The curves are plotted for $H=-0.2$ (blue) and $H=0.4$ (red). (d) Bifurcation diagram for $\beta=2.5$. For a set of parameters there are three fixed points, but if the external field is too strong (in absolute value), two of the fixed points disappear in a saddle-node bifurcation. (e) Bifurcations in two-parameter plane. For $H=0$ and $\beta=1$ there is a cusp.

that point, a symmetry breaking occurs (assuming tiny noise in the system or slightly asymmetric initial conditions). However, the pitchfork bifurcation is structurally unstable with respect to the second control parameter $H$ : for nonzero $H$, saddle-node bifurcations occur instead [see Figs. 1(c) and 1(d)]. The system has thus always either one or three fixed points. In the latter situation, two out of three fixed points are always stable and for $\beta=1$ and $H=0$ one can observe a cusp [Fig. 1(e)]. 


\section{Extended Mean-Field Ising Model}

We generalize map (2) by introducing a dynamics on the field $H$. In the standard map (2), as already mentioned, $H$ is considered to be exogenous, and is taken to represent the influence of outside news on the opinion or decision $s(t)$ of the typical agent. Motivated by models of financial price dynamics Kaizoji et al., 2015; Ollikainen, 2016], we propose to interpret $H$ as a measure of momentum of the opinion dynamics. To reduce the dynamics to arguably the simplest model without the need for specifying the price evolution, one can consider that the opinion dynamics momentum is a proxy for the price momentum included in the asset price dynamics of Kaizoji et al., 2015]. Let us recall that the logic of the initial model Kaizoji et al., 2015] is that the so-called noise traders or technical analysts come to their investment decisions based on the information they gather from the opinions of their fellow noise traders and on their measure of the strength of the price trends. Trend-following or momentum investing is indeed a widely used class of investment strategies (see e.g. Ross et al., 2017; Lempérière et al., 2014; Roncalli, 2017]). The influence of the decisions of other investors is captured by the term $\beta s_{t}$ in (2). The momentum of the social opinion mirroring the trend of the price is embodied in the $H$ term, which is assumed to be given by the following auto-regressive dynamics

$$
H_{t+1}=\theta \cdot H_{t}+(1-\theta) \cdot s_{t}, \quad \text { where } \theta \in[0,1) .
$$

Equation (3) defines $H_{t}$ as the exponential moving average of approximately $n_{\theta} \simeq 1 /(1-\theta)$ previous opinion states $\left\{s_{t-1}, s_{t-2}, \ldots, s_{t-n_{\theta}}\right\}$. For $\theta=0$, Eq. (3) gives $H_{t+1}=s_{t}$, corresponding to a one-step memory. For $\theta \rightarrow 1^{-}$, the memory becomes infinite (with $H_{t+1}=H_{t}=H_{0}$ ) and the initial momentum value $H_{0}$ is always remembered.

Putting Eq. (3) together with Eq. (21) yields the dynamical system that we study in this article,

$$
\left\{\begin{array}{l}
s_{t+1}=\tanh \left(a \cdot s_{t}+b \cdot H_{t}\right), \\
H_{t+1}=\theta \cdot H_{t}+(1-\theta) s_{t},
\end{array}\right.
$$

where $s_{t}$ is the opinion (positive - buy, negative - sell) of the representative investor and $H_{t}$ is its momentum. For $\theta=0$, this system reduces to the dynamical mean-field Ising model (2) for $a=b=\beta$. For notational convenience, we will refer to the 2D-map (4) as $M$, such that $\left\{s_{t+1}, H_{t+1}\right\}=$ $M\left(\left\{s_{t}, H_{t}\right\}\right)$.

In the standard mean-field equation (11), and by extension in the map (2), the parameters $a$ and $b$ are usually taken to be positive, corresponding respectively to so-called "ferromagnetic" interactions (or positive feedback and imitation in the social context) and a positive organizing effect of the external influence $H$. Negative values of $a$ should not be interpreted as "antiferromagnetic" interactions, since the latter refer to a propensity to take a spin value opposite to that of the neighbor, which is different from a tendency to take the sign at the next time step that is the opposite of the sign at the previous time step. Instead, a negative value of $a$ in (4) can be interpreted as a contrarian behavior tending to correct at the next time step what can be perceived as a dangerous consensus. Such a mechanism was identified and explored by Corcos et al. 2002. in a simpler 1D-map describing the proportion of "bullish" agents in the population of investors. The rationale for negative values of $a$ is thus that investors may become worried when the consensus is too large, which may signal an exuberant unsustainable bubble. In response, these investors may decide to become contrarians and change their decision. If the representative agent adopts this stance, this will give oscillatory dynamics as well as deterministically chaotic behaviors, as we document in detail below. Similarly, negative values of parameter $b$ can be rationalized by such a contrarian response, but now built on a longer time scale according to the sensitivity of a growing trend.

\section{Bifurcations for Fixed $\theta$}

Let us explore the transitions that can be observed in the system (41) for certain fixed values of $0<$ $\theta<1$. The Jacobian $J$ and its eigenvalues $\lambda_{1}$ and $\lambda_{2}$ are

$$
J=\left[\begin{array}{cc}
a & b \\
1-\theta & \theta
\end{array}\right]
$$

and

$$
\lambda_{1,2}=\frac{a+\theta \pm \sqrt{(a-\theta)^{2}+4 b(1-\theta)}}{2} .
$$

\subsection{Codimension-1 bifurcations}

The following bifurcations of codimension-1 can be classified. 
(1) Bifurcations of the fixed point $(0,0)$ :

(a) A pitchfork bifurcation occurs when one of the eigenvalues is equal to 1 (marked with the black solid line in the two-parameter bifurcation diagram in Fig. 3 and with the black surface in Fig. 4). The bifurcation occurs for $a$ obeying the following dependence as a function of $b$ and $\theta$ :

$$
a(b, \theta)=-b+1 .
$$

(b) Period doubling (flip) occurs when one of the eigenvalues is equal to -1 (marked as the red solid line in the two-parameter bifurcation diagram in Fig. 3 and as the red surface in Fig. (4). The bifurcation occurs for $a$ obeying the following dependence as a function of $b$ and $\theta$ :

$$
a(b, \theta)=b \frac{1-\theta}{1+\theta}-1 .
$$

(c) Neimark-Sacker bifurcation occurs when both eigenvalues lie on the unit circle and have equal real parts (marked as a green solid line below the black one in Fig. 3 and with the green surface in Fig. (4). The bifurcation occurs for $a$ obeying the following dependence as a function of $b$ and $\theta$ :

$$
a(b, \theta)=\frac{1+b(1-\theta)}{\theta} .
$$

(2) Bifurcations of nonzero fixed points (there is no available analytical expression):

(a) Period doubling (as previously; marked with light blue line in Fig. (3).

(b) Neimark-Sacker bifurcation (as previously; marked with green solid line above the black one in Fig. 3).

(3) Bifurcations of period-2 fixed points (no explicit analytical expression):

(a) Pitchfork bifurcation occurs when the period-2 fixed point has one eigenvalue equal to 1 under the twice iterated map $M(M(\cdot))$. It is marked with the blue solid line in the two-parameter bifurcation diagrams.

(b) Neimark-Sacker bifurcation (as previously but for the second iterate of the map $M$, namely $M(M(\cdot)))$.

Figure 2 presents bifurcation diagrams for one value of $\theta=0.99$ and three different values of $b$,

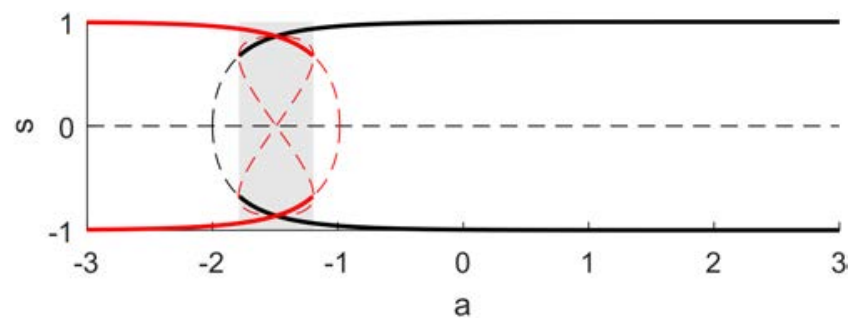

(a)

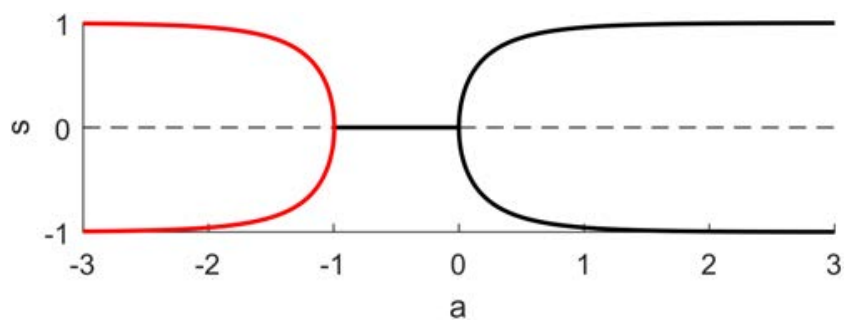

(b)

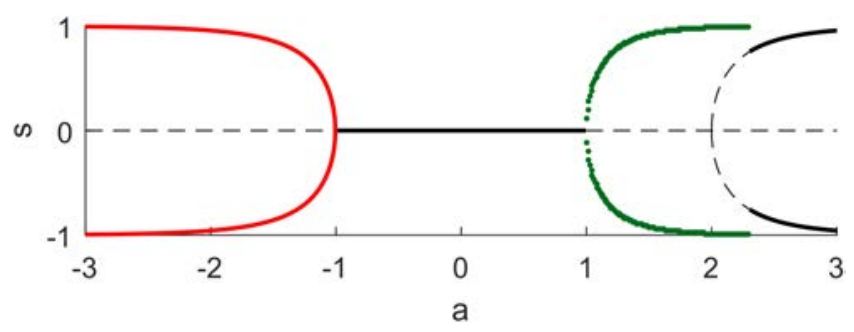

(c)

Fig. 2. One-parameter bifurcation diagrams for $\theta=0.99$. The solid lines stand for stable points and dashed lines for unstable. The colors black, red and green represent fixed points, period-2 fixed points and oscillatory or quasioscillatory behavior, respectively. (a) $b=3$, (b) $b=1$ and (c) $b=-1$.

namely 3,1 and -1 . In all the cases, for large values of $a$ there are stable nontrivial fixed points (black solid) and, for small values of $a$, there are stable period-2 points (red solid). Depending on $b$, the transition between those states occurs in a variety of ways. For $b=3$ [Fig. 2(a)] while varying $a$, the following bifurcations occur (from left to right): pitchfork giving rise to two new unstable fixed points; period doubling of unstable fixed points; pitchfork of period-2 fixed points; period doubling of unstable trivial fixed point. The region of bistability between flipping (jumping from one period-2 fixed point to another) and nonzero fixed points is shown in gray shade. Decreasing $b$ to 1 [Fig. 2(b)] shifts linearly the pitchfork bifurcation to the right in terms of $a$ [see Eq. (7)], whereas the flip remains practically in the same position $\left[\frac{1-\theta}{1+\theta} \approx 0\right.$ in Eq. (8) $]$. This leads 


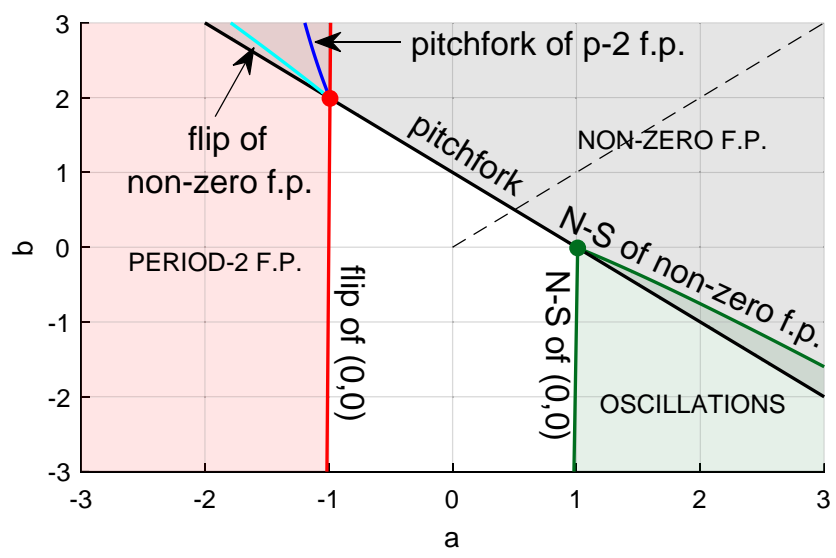

(a)

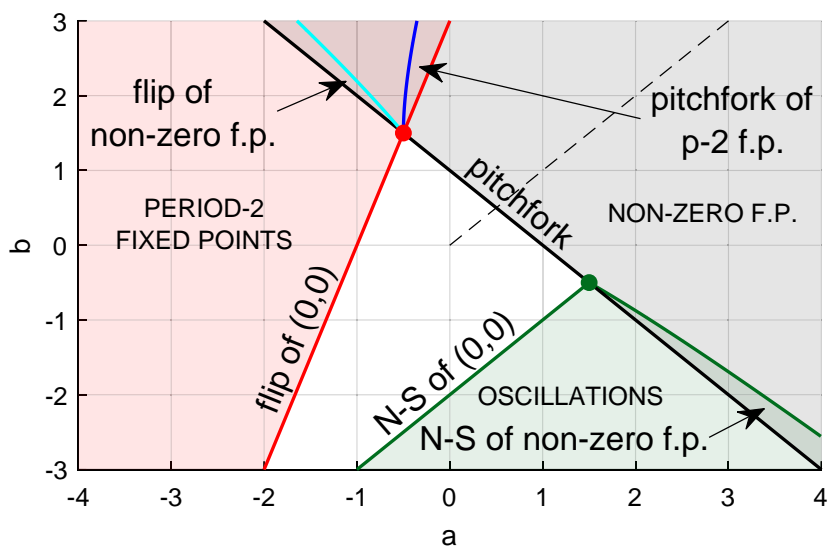

(b)

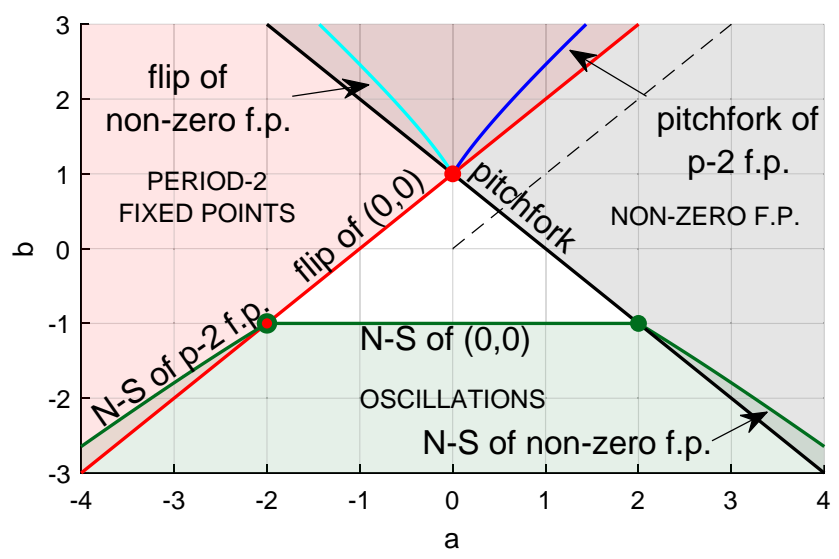

(c)

Fig. 3. Bifurcation diagrams of system (4) in the two-parameter plane $\{a, b\}$ for three fixed values of $\theta$. (a) $\theta=0.99$, (b) $\theta=0.50$ and (c) $\theta=0.00$. Beside all the bifurcation curves, the black dashed lines represent the case $a=b=\beta \geq 0$, corresponding to the original 1D-map (2). Similarly to the situation shown in Fig. 1(b), a pitchfork bifurcation also occurs here, but for $\beta=0.5$ (instead of $\beta=1$ ), due to the contribution of the momentum term in 44). Scanning all possible values of $a$ and $b$, a variety of behaviors can be classified. All the diagrams for different values of $\theta$ are qualitatively similar. As can be deduced from Eq. (7), $\theta$ does not influence the position of the pitchfork bifurcations at all. The other two bifurcations (period doubling - red, Neimark-Sacker - green) of the trivial fixed points move as $\theta$ varies. For the singular case of $\theta=1$, these bifurcation lines become parallel. The domain in white represents the existence of only one attractor, which is the trivial equilibrium.

to "untying" the characteristic 8-shaped curve and leaves only bifurcations of the trivial fixed point. For $b$ becoming sufficiently negative [here: $b=-1$ in Fig. 2(c)], additional phenomena occur. At $a \approx 1$, there is a Neimark-Sacker bifurcation that destabilizes the trivial fixed point giving rise to stable oscillations. The oscillations disappear due to a sequence of bifurcations in a tiny region in parameter space (for more information see: Chapter 7 in [Guckenheimer \& Holmes, 1983]). Bistability can be found there as well, but the region is so narrow as to be invisible.

\subsection{Codimension-2 bifurcations}

For the two-dimensional discrete system (4), codimension-2 bifurcations occur if $\left|\lambda_{1}\right|=\left|\lambda_{2}\right|=1$. This implies that there are three possible bifurcations and all of them can be parametrized in terms of $\theta$ :

(1) $\lambda_{1}=\lambda_{2}=1$ (pitchfork + Neimark-Sacker; green points):

$$
\begin{aligned}
& a=-\theta+2, \\
& b=\theta-1 .
\end{aligned}
$$




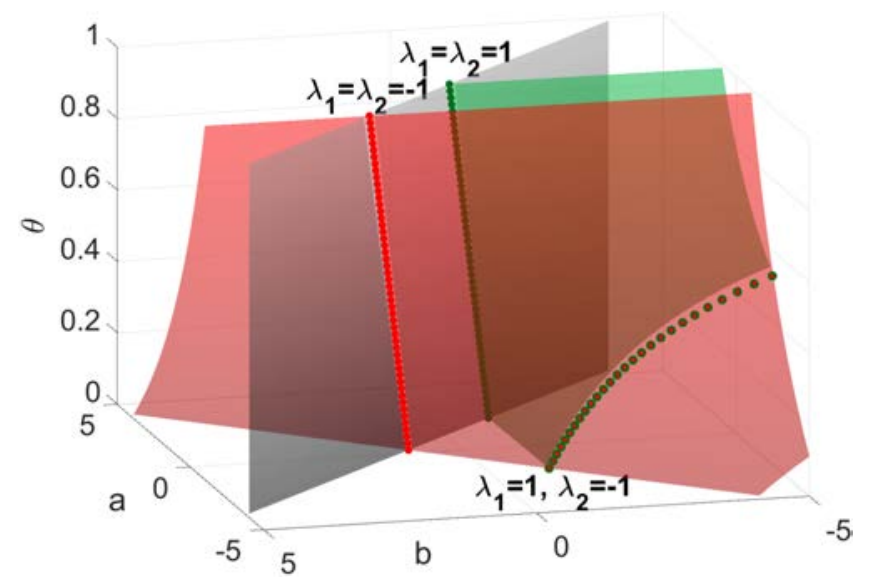

Fig. 4. Scan of the values of $\theta$ for codimension-1 and -2 bifurcations of the fixed point $(0,0)$. The surfaces in black, red and green correspond to the boundaries for the pitchfork, flip and Neimark-Sacker bifurcations, respectively.

(2) $\lambda_{1}=1$ and $\lambda_{2}=-1$ (pitchfork + flip; red points):

$$
a=-\theta, \quad b=\theta+1 .
$$

(3) $\lambda_{1}=\lambda_{2}=-1$ (flip + Neimark-Sacker; green points with red face color):

$$
a=-\theta-2, \quad b=\frac{(\theta+1)^{2}}{\theta-1} .
$$

We present the curves where codimension-2 bifurcations occur as bold points both in Fig. 3 and also in the three-dimensional plane $\{a, b, \theta\}$ in Fig. 4. Figure 4 visualizes additionally codimension1 bifurcations for which the analytical form exists. One notices that for $\theta=0$ codimension- 2 bifurcations occur close to each other, whereas for $\theta \rightarrow 1$ one of those bifurcations is shifted towards infinity.

\section{Comparison Between Extended and Original Mean-Field Ising Models}

The crucial question to be answered at this point is how the behavior of the extended system actually varies from what was observed in the original Ising mean-field model. If $a=b=\beta \geq 0$, the twodimensional system, independently of $\theta$, exhibits a bifurcation at $\beta=0.5$. In contrast to the system (2) and its pitchfork in $\beta=1$, adding the equation for $H_{t+1}$ in (4) makes the bifurcation occur for a value $\beta$ twice smaller. This is caused by the fact that, around the stable fixed point $(0,0)$, we can use the approximation $s_{t} \approx H_{t}$ and retrieve $s_{t+1}=\tanh \left[2 \beta\left(s_{t}+0\right)\right]$.

If the system (4) is constrained to the physical interpretation of the parameters, i.e. $a>0$ and $b>0$, and if the memory parameter $\theta$ is less than 1 , there always exists a region where the period-2 solutions arise. This follows from Eq. (8), $a(b, \theta)=b \frac{1-\theta}{1+\theta}-1$, governing the position of the flip bifurcation. Obviously, for any given positive $\theta<1$, the term $\frac{1-\theta}{1+\theta}$ is positive as well. Therefore, one can find a sufficiently large $b$ such that $b \frac{1-\theta}{1+\theta}>1$, hence there always will exist $a>0$ where period-2 points exist. Nevertheless, this does not mean that those periodic points are directly observable as they

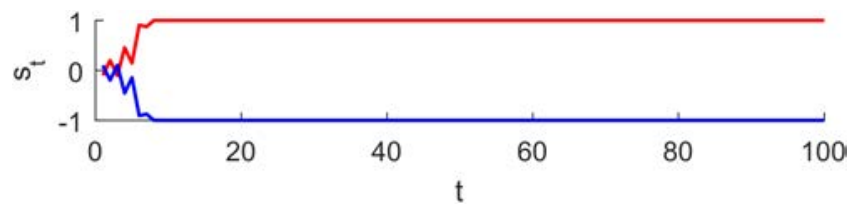

(a)

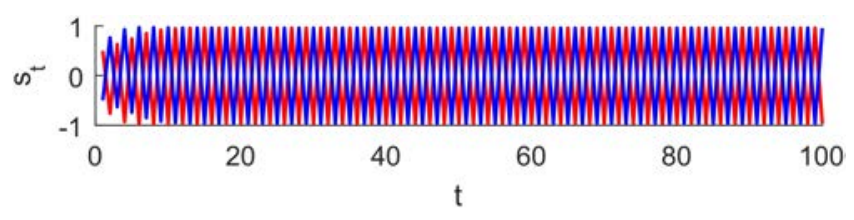

(b)

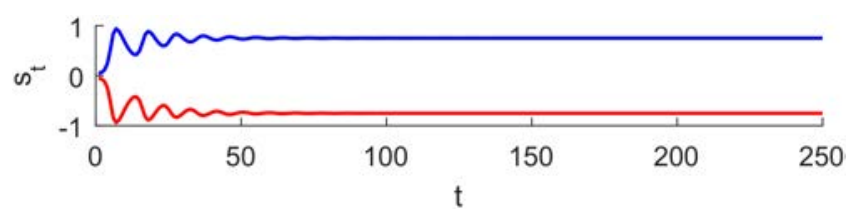

(c)

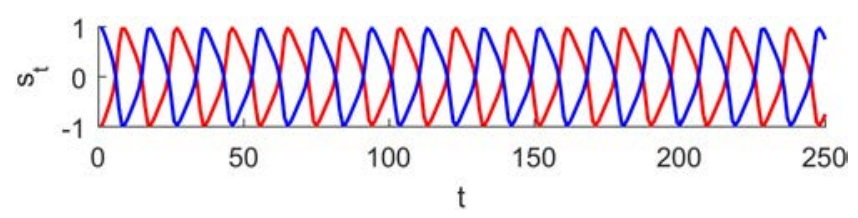

(d)

Fig. 5. Time profiles for different initial conditions and $\theta=0$. (a) and (b) $a=1, b=3$. Initial conditions: (a) in red: $s_{0}=-0.1$ and $H_{0}=0.1$, in blue: $s_{0}=0.1$ and $H_{0}=-0.1$, (b) in red: $s_{0}=0.5$ and $H_{0}=-0.5$, in blue: $s_{0}=-0.5$ and $H_{0}=0.5$. (c) and (d) $a=3.3, b=-2$. Initial conditions: (c) in red: $s_{0}=-0.05$ and $H_{0}=-0.05$, in blue: $s_{0}=0.05$ and $H_{0}=0.05$, (d) in red: $s_{0}=-0.99$ and $H_{0}=-0.99$, in blue: $s_{0}=0.99$ and $H_{0}=0.99$. 
are not stable until the pitchfork of period-2 point occurs. This bifurcation is marked with dark blue color in Fig. 3 but also occurs at the right boundary of the shaded interval in Fig. 2(a). When the period-2 points stabilize, one can observe that, for different initial conditions, the system might behave differently - it might either converge to a single fixed point (positive or negative one) or to a flipping behavior [see Figs. 5(a) and 5(b)].

Numerical simulations show that, even for $\theta$ very close to 1 , the curve of period-2 fixed point pitchfork bifurcation (dark blue line in Fig. 31) crosses the line $a=0$. We present in Fig. 6 the values of $b$ when $a$ crosses 0 for certain values of $\theta$.

To sum up what was stated above, we would like to underline that, for different values of $a>$ 0 and $b>0$, only three types of attractors are possible:

- trivial equilibrium,

- nontrivial fixed point (positive and negative),

- period-2 fixed points.

If we allow $a$ and $b$ to take values from the whole $\mathbb{R}^{2}$, the system may exhibit a much broader variety of behaviors. There is for instance a set of parameters for which oscillations occur (see the green filled

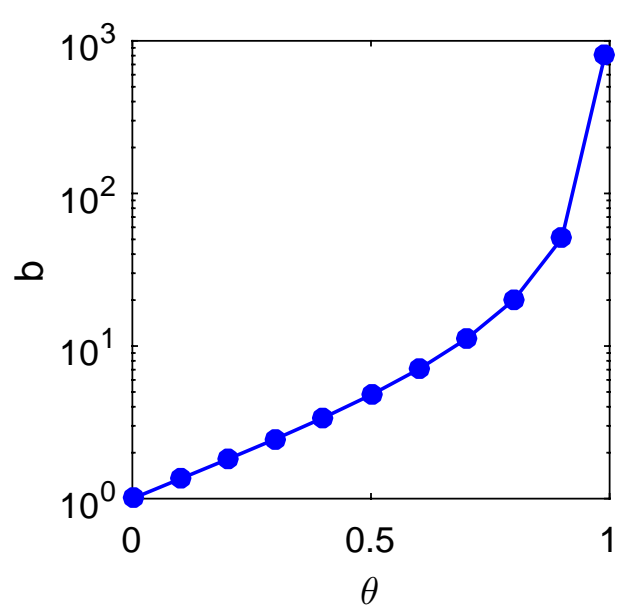

Fig. 6. Value of $b$ for $a=0$ on the dark blue curve representing pitchfork bifurcations to period-2 fixed points depending on $\theta$. The selected points represent where the bistability occurs in the regime $b>0$. The diagram says that for every $\theta<1$ there occurs bistability in the physically justified region $(a>0, b>0)$. Of course, for $\theta$ near 1 , this region might not be accessible as $b$ needs to be very large. For lower values of $\theta$, the parameter $b$ decreases gradually to 0 . This means that the lower the memory coefficient, the lower parameter $b$ needs to obtain bistability in the physical region of positive parameters $a$ and $b$. area in Fig. 3). The region of existence of oscillations intersects with the region where the nontrivial stable solutions exist [see Figs. 5(c) and 5(d)]. We do not explore it further here, but some of the oscillations can be quasi-periodic, while others stay strictly periodic. We have observed periodic behavior for periods between 3 and 8 (we assume that higher periodicity is also possible), and even chaotic behavior. The latter is analyzed further in Sec. 6

\section{Chaos}

This section presents several tests to show where, both in time space and in parameter space, chaos exists and what kind of attractors can be expected in system (4).

In order to better understand the bifurcations present in the system, we explore the largest Lyapunov exponent for several values of $\theta$, computed by examining the growth of solutions of the variational equation for a typical initial condition and perturbation vector. The result is presented in Fig. 7: blue denotes negative and red denotes positive exponents. For $a>0$ and $b>0$ we find no chaos, but for some negative values of $a$ or $b$, more interesting behaviors occur. The diagrams in Fig. 7 clearly show many features of the bifurcation diagrams in Fig. 3 - in particular the lines of bifurcation of stable attractors. The bifurcations of unstable attractors are however not visible. Note that in order to compensate for the slow dynamics as $\theta \rightarrow 1$, we normalize by $(1-\theta)$.

In Fig. [7(a) $(\theta=0.99)$ the slightly visible light red dots scattered in the right part of the diagram correspond to quasi-periodic behavior, much more iterations taken into computation of Lyapunov exponent will allow them to vanish. For $\theta=0.00$ [Fig. [7(c)], we have been unable to identify any trace of chaos within the selected parameter values. Analysis of Fig. [7(b) $(\theta=0.5)$ suggests that the parameter domain $a \in(-5,-3)$ and $b \in(-10,-8)$ may be the most favorable to find chaotic behavior. Therefore, we explore below this region more thoroughly.

For example, let us fix $a=-4.17$ and $\theta=0.5$. These parameters are chosen so as to obtain several regions of chaotic behavior while scanning $b$. Figure 8 (a) illustrates the attractors as the $s$ component against parameter $b$. To generate this kind of diagram, we start with two initial conditions $\{-0.5,0.5\}$ and $\{0.5,-0.5\}$ for $b=-10.3$ and iterate the system 400 times. Then, we discard the 


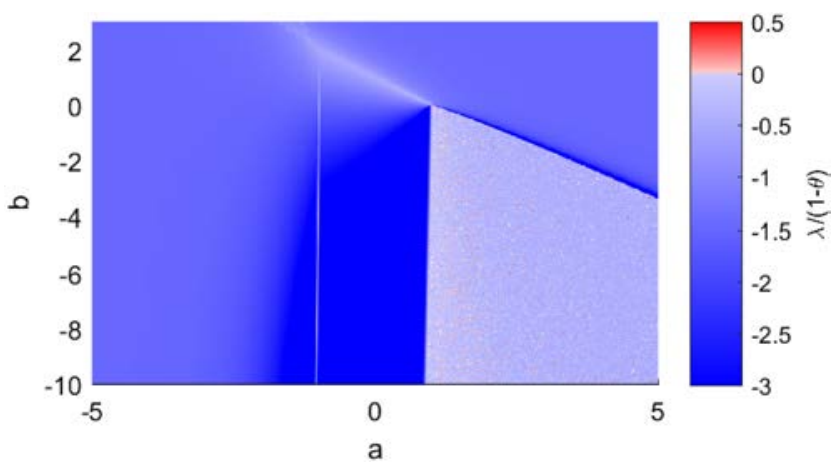

(a)

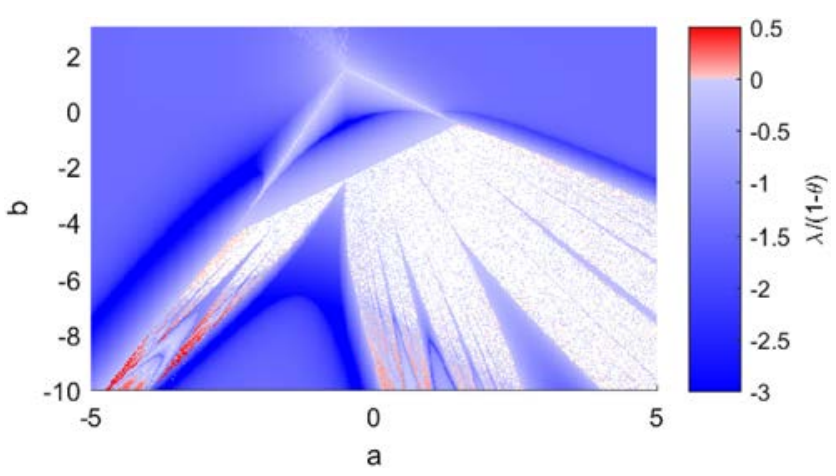

(b)

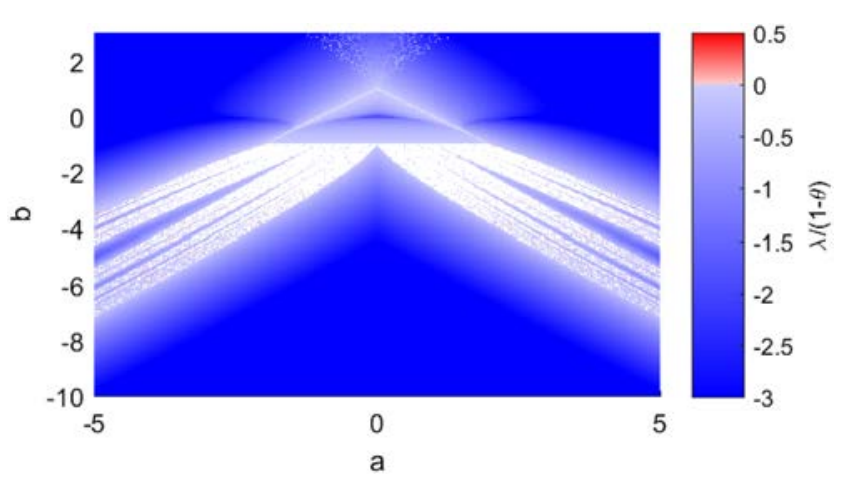

(c)

Fig. 7. Largest Lyapunov exponent, rescaled by $\frac{1}{1-\theta}$. (a) $\theta=0.99$, (b) $\theta=0.50$ and (c) $\theta=0.00$. The parameters are chosen so as to correspond with Fig. 3 For $\theta=0.99$ [panel (a)] there is no region with chaotic behavior, the sporadic light red dots in the bottom right-hand side of the plot are caused by quasi-periodic behavior and cannot be interpreted as chaos. In panel (b) one can observe a region with potentially chaotic behavior, moreover, in panels (b) and (c) there are characteristic Arnold tongues corresponding to period locking.

first 100 points and plot the last 300 points. For the next value of $b$, namely -10.29 , we use as the initial condition the final state of the system after those 400 iterations. The procedure is repeated until $b=-8.3$. The attractors obtained in this scheme are plotted in black and blue. Moreover, we perform the procedure identically, starting with the same initial conditions and decreasing $b$ from -8.3 to -10.3 . The attractors are then plotted in green and red. It is visible that changing the direction of the scan helps to discover different attractors and the system is multistable or at least bistable in the vast part of the diagram - up to $b \approx-8.5$.

For all the attractors, we investigate again the Lyapunov exponent to identify where exactly the chaotic attractors can be expected [see Fig. 8(b)].

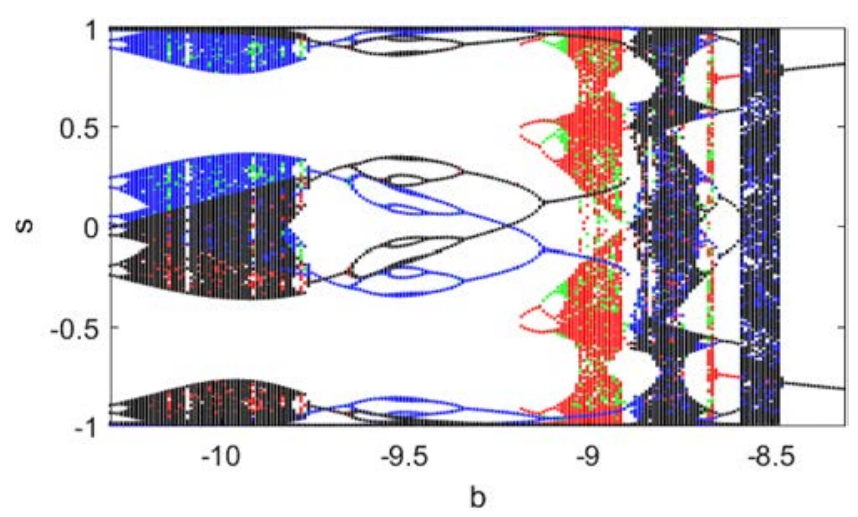

(a)

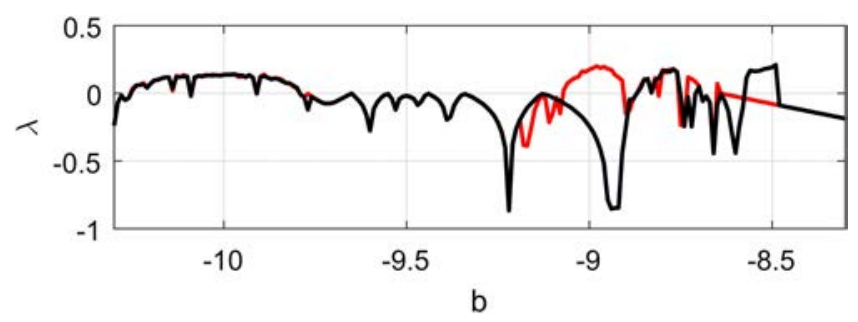

(b)

Fig. 8. Bifurcation diagrams illustrating multistability and chaotic behavior in the system (4. For both panels, the parameters are $a=-4.17$ and $\theta=0.5$. (a) Bifurcation diagram of system (4). In red and blue are marked attractors to which the system converges when increasing $b$ and initiated in $(-0.5,0.5)$ and in $(0.5,-0.5)$ respectively. Similarly, for decreasing $b$, we use green and red colors. One can deduce that chaotic behavior is possible within several intervals $(b$ around -10 , around -8.75 and around -8.55$)$. For other values of $b$, the system converges to periodic or quasi-periodic oscillations. For $b=-9$, there are two period-3 orbits coexistent with a chaotic attractor, whereas for $b=-9.5$ one can observe two separated period-24 orbits. In between, there is a triple cascade of period-doubling bifurcations. (b) The largest of the two Lyapunov exponents for the blue attractor in A. Occurrences of Lyapunov exponent $\lambda>0$ confirm that one can expect chaos for $b \approx-10, b \approx-9, b \approx-8.77$ and for $b \approx-8.53$. 


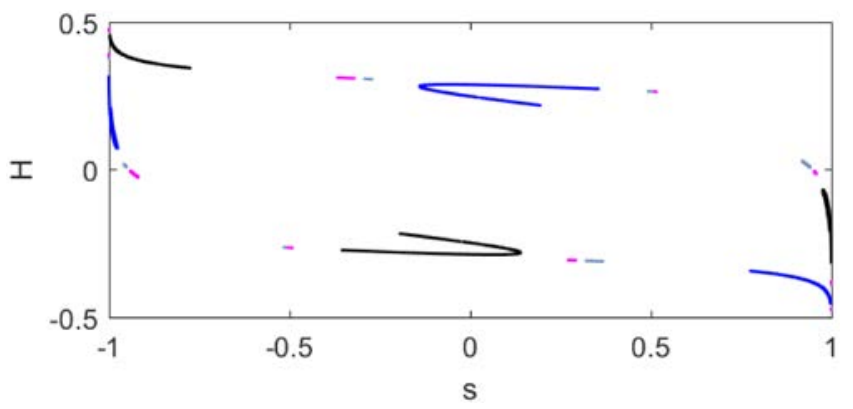

(a)

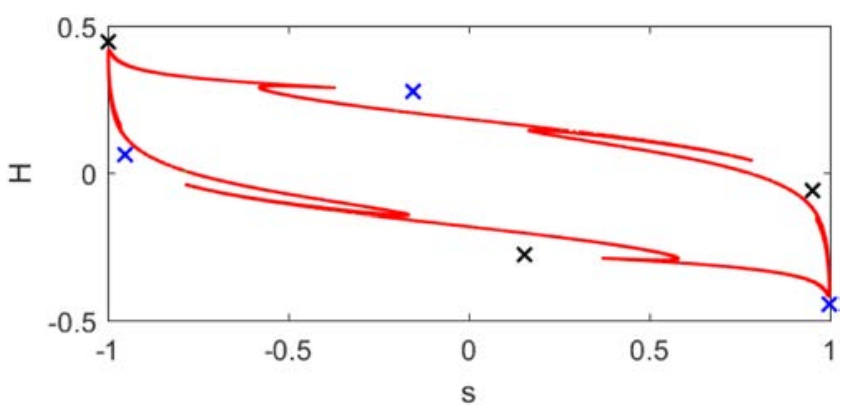

(b)

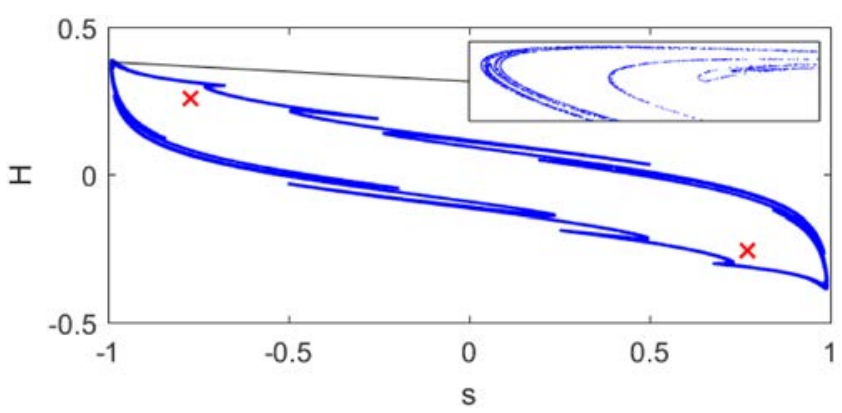

(c)

Fig. 9. Examples of chaotic attractors for $a=-4.17$ and $\theta=0.5$ : (a) Shows four simultaneously stable chaotic attractors for $b=-10$, (b) shows a single chaotic attractor (red) simultaneously stable with two period-3 orbits (blue, black) for $b=-9$, and (c) shows bistability between a single chaotic (blue) and a periodic (red) attractor for $b=-8.53$. The colors correspond to the colors in Fig. 8 1 a). Observe the characteristic folding of the chaotic attractors.

Indeed, there are several regions with a positive exponent. We select some of them and plot the attractors in phase space $\{s, H\}$ in Fig. 9. The methodology explained above obviously does not provide all the attractors, which can be instantly spotted in Fig. 9(a), where two minor attractors are marked.

The attractors in Figs. 9(b) and 9(c) are more interesting. When zoomed-in on the tip of the

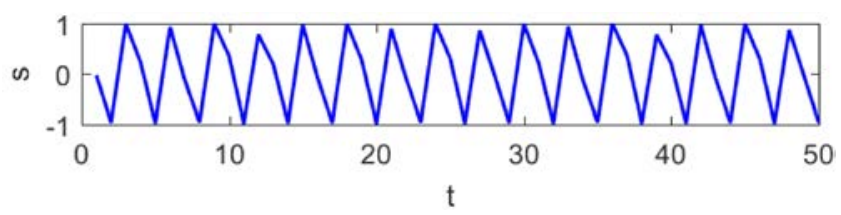

(a)

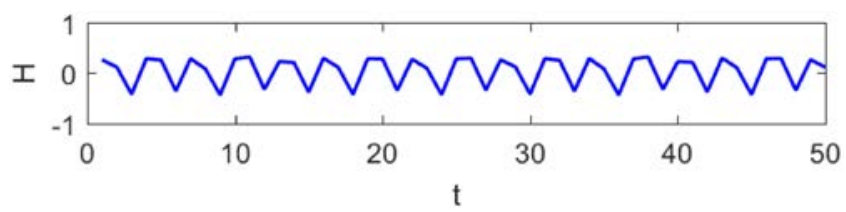

(b)

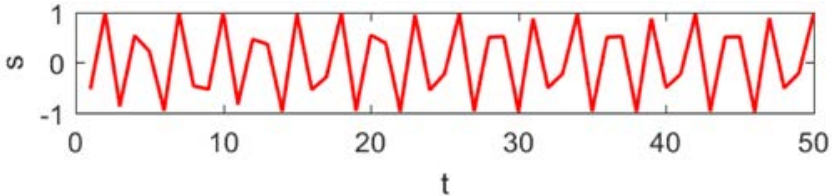

(c)

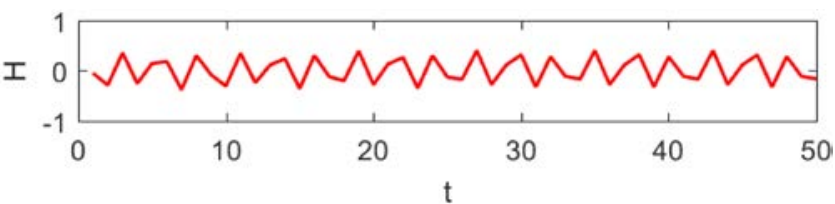

(d)

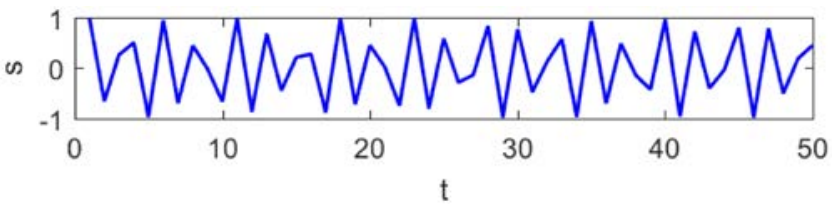

(e)

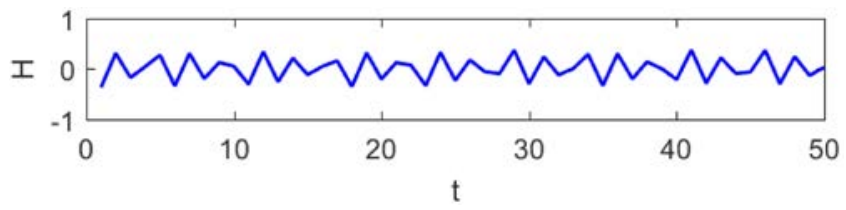

(f)

Fig. 10. Chaotic trajectories corresponding to the attractors presented in Fig. 9. (a) $s$ variable taken in the blue region in Fig. 9(a), (b) $H$ variable taken in the blue region in Fig. 9(a), (c) $s$ variable taken in the blue region in Fig. 9 b), (d) $H$ variable taken in the blue region in Fig. 9(b), (e) $s$ variable taken in the blue region in Fig. 9(c) and (f) $H$ variable taken in the blue region in Fig. 9 (c). 
attractor, we can clearly see the characteristic chaotic folding. This allows us to conclude that, indeed, chaotic behavior is possible in the dynamics of noise traders' opinions.

To provide basic intuitions about the system's behavior in the chaotic regimes, we include Fig. 10 with time series of $s$ and $H$ for some of the attractors presented in Fig. 9. The colors of the time series correspond to the colors of the chaotic attractors.

\section{Market Passages Through a Bifurcation}

This section presents how parameter changes can influence the noise traders' behaviors. We analyze four scenarios of parameter shifts across certain bifurcation curves. To keep it simple, we vary only parameter $a$ fixing $b$ and $\theta$. One can interpret the linearly shifted parameter as an extension of the system (44) taking the form:

$$
\left\{\begin{array}{l}
s_{t+1}=\tanh \left(a_{t} \cdot s_{t}+b \cdot H_{t}\right), \\
H_{t+1}=\theta \cdot H_{t}+(1-\theta) s_{t}, \\
a_{t+1}=a_{t}+k,
\end{array}\right.
$$

where $k$ is a fixed value.

Let us analyze four scenarios:

(1) Market passage through flip and NeimarkSacker bifurcation [Fig. 11( $a), k=0.04]$. This scenario begins with a situation where the traders have mixed stable opinions whose average is $s_{t}=0$. Let us then pick an average memory length $(\theta=0.5)$ and contrarian attitudes $(a=-1$ and $b=-2)$ representing for instance mindsets after a recent financial draw-down. This is a regime with only one stable state. If the market conditions change in terms of parameter $a$ (for instance to a more contrarian stance $-a=-3$, or to a highly imitating behavior $-a=3$ ), the traders' opinions are destabilized and the variables start to flip or oscillate. Two important observations can be made at this point:

(a) if the market conditions change because of reasons which are difficult to track, it might not be possible to determine which way the parameter $a$ should be changed to revert to the original state;

(b) a transition back to the nominal state $s_{t}=0$ requires a larger shift of $a$ than the original one. For instance, if a shift occurs from a fixed point

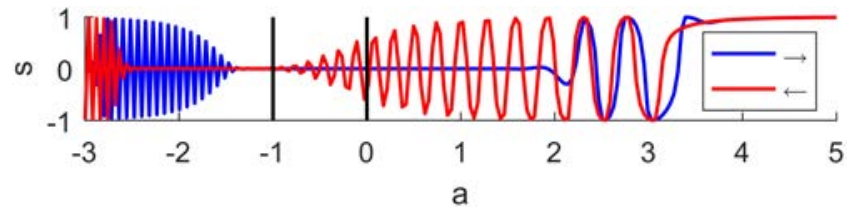

(a)

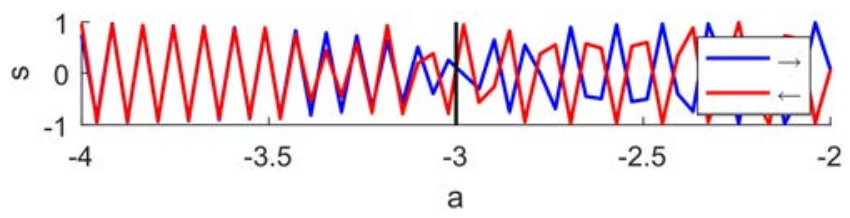

(b)

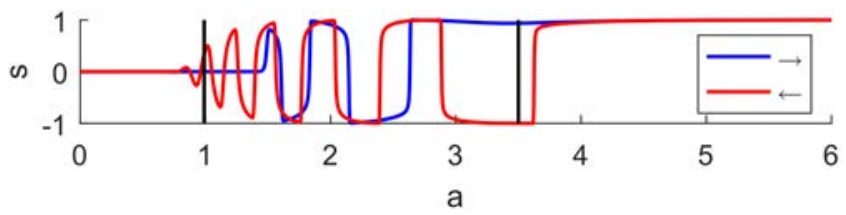

(c)

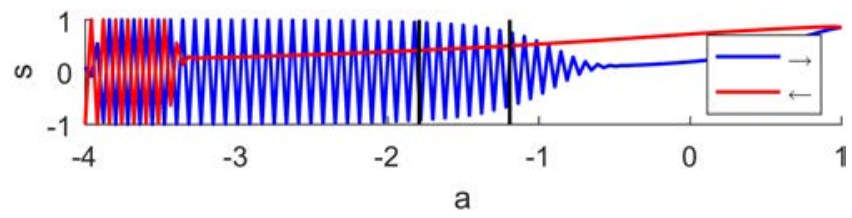

(d)

Fig. 11. Linear passages through bifurcations as described by the system (13). Initial values are taken in the neighborhood of an attractor corresponding to the parameter $a$ given by the leftmost value in each diagram. Parameter $a$ is increased at each time iteration by a certain fixed value $k$, leading to the dynamics for the order parameter represented by the blue curve. The red curve is generated by decreasing $a$ from the rightmost value. (a) $\theta=0.50, a \in[-3,5], b=-2$, $k=0.04$, (b) $\theta=0.50, a \in[-4,-2], b=-6, k=0.04$, (c) $\theta=0.99, a \in[0,6], b=-2, k=0.004$ and (d) $\theta=0.99$, $a \in[-3,0], b=3, k=0.04$. Black vertical lines represent where the bifurcations occur.

with $a=1$ to $a=3$ (blue trajectory), oscillations appear. A backward transition to a state without oscillations (red trajectory) requires shifting the parameter much further than just to 1 , namely as far as $a=-1$. A similar behavior exists when decreasing $a$ from $a=-2$ to $a=-3$ (red trajectory) and then increasing $a$ (blue trajectory) to -1 . This might be confused with a hysteresis loop, but this is actually a bifurcation delay that arises often in fast-slow dynamical systems (see for instance Baesens, 1995] or [Mandel \& Erneux, 1984]). 
(2) Market passage to a chaotic regime [Fig. 11](b), $k=0.04]$. When the system is in a highly contrarian flipping state $(\theta=0.5, a=-4, b=-6)$, certain parameter changes might lead to a transition to a chaotic regime. Then, instead of switching opinions at every step, the behavior of traders cannot be predicted, which might lead to an undesired market behavior. Nevertheless, there is some remaining regularity in the sense that, in the chaotic regime, one can observe that the transition from a positive to a negative opinion state and vice versa occurs at least once per three steps. The bifurcation delay is not significant in this scenario, after 3-4 iterations when moving leftwards through the bifurcation line the system retrieves its flipping behavior.

(3) Market passage into the rapid oscillations regime [Fig. 11(c), $k=0.004]$. Similarly to the first scenario, the transition through a Neimark-Sacker bifurcation of the trivial fixed point exhibits a bifurcation delay. Moreover, the oscillations become very rapid and not symmetric (see Chapter 3 in Ollikainen, 2016]), which corresponds to large market price changes such as during bubbles and crashes. Decreasing parameter $a$ can lead to accelerating oscillations (such as those described by the socalled log-periodic power law singularity (LPPLS) models presented for instance in Johansen et al., 1999, 2000; Sornette, 2003; Sornette et al., 2013]). On the other hand, the system can pass through another Neimark-Sacker bifurcation (this time of nonzero fixed points) and stabilize on a nontrivial positive or negative fixed point. The way the system settles down on one of the fixed points is very sensitive to the current opinion value. This means that, if the market opinion varies quickly, it is prone to settle easily to either a "bullish" or a "bearish" market, depending not only on the parameter value $a$, but also on the transition time as well. This may lead to market unpredictability even if all the parameters could be known precisely.

(4) Switching through a bistable region [Fig. 11](d), $k=0.04]$. The last scenario we present is a passage across four bifurcation curves, but actually only two of them change the behavior of the system. Those are the flip of nonzero fixed points and the pitchfork of period- 2 fixed points. The region in the middle is bistable as in Fig. 2(a). The behavior of the system is in essence a mixture of three components: (a) bifurcation delay as in scenario 1, (b) sensitivity of settling down as in scenario 3, and (c) hysteresis in response (see for instance the saddle-node case in Kuznetsov, 1998). Component (c) is caused by the existing bistability. Namely, for lower values of $a$, the system jumps up and down. After the second black line in Fig. 11(d), it slowly converges to a fixed point. On the way back, it starts to flip very slowly when the system passes the left black line. This hysteresis in behavior results in a much larger delay than observed in scenario 1 . Also, if the system is on the fixed point in the bistable region and $a$ is decreased far enough, in order to retrieve the fixed point, reverting the parameter to its original state might not be sufficient to suppress the flipping. Economically speaking, this means that, if there is a small change which destabilizes the market, simply reverting the change might not be enough to stabilize the system again and thus much larger interventions might be needed.

\section{Conclusions}

We have introduced and analyzed in detail an extended two-dimensional dynamical version of the mean-field Ising model. Inspired by the dynamics of social imitation in financial markets involving fast imitation and slower trend following, the traders' opinion dynamics is modeled as the interplay between instantaneous social imitation and past trends in social coordinations. The standard magnetic field in the Ising model is reinterpreted and endogenized as a smoothed moving average of the past state opinion variable.

We have shown the existence of a rich set of bifurcations as a function of the two parameters $a$ and $b$ quantifying the relative importance of instantaneous versus past social opinions on the formation of the next value of the state variable. The dependence as a function of a third parameter $\theta$ controlling the memory length over the past states has also been dissected. We have presented a thorough analysis of the existence of chaotic behavior, present in certain parameter regimes. Finally, we have examined four scenarios in which a slow change of a control parameter induces transitions through bifurcation boundaries. These scenarios have been offered as possible simplified models of changes of regimes in financial markets. One important lesson is that, due to the phenomena of delayed bifurcations often associated with fast-slow dynamical systems as well as of possible hysteresis, the amplitude of the corrections needed to recover from a crisis and to push the system back to "normal" may be significantly 
larger than the strength of the causes that led to the crisis itself. In other terms, this is a quantitative reminder that "prevention is better than cure", at least in our conceptual model of financial market opinion dynamics.

\section{Author Contribution Statement}

The manuscript was mostly prepared by the first author, based on research directed by all the authors. All the authors have read and approved the final manuscript.

\section{Acknowledgments}

The authors are grateful for the opportunity to work within the CRITICS Innovative Training Network. This project has received funding from the European Union's Horizon 2020 research and innovation programme under the Marie Skłodowska-Curie grant agreement No. 643073.

\section{References}

Baesens, C. [1995] "Gevrey series and dynamic bifurcations for analytic slow-fast mappings," Nonlinearity 8, 179-201.

Brock, W. A. \& Durlauf, S. N. [2001] "Discrete choice with social interactions," Rev. Econ. Stud. 68, 235260.

Callen, E. \& Shapero, D. [1974] "A theory of social imitation," Phys. Today 27, 23-28.

Corcos, A., Eckmann, J.-P., Malaspinas, A., Malevergne, Y. \& Sornette, D. [2002] "Imitation and contrarian behavior: Hyperbolic bubbles, crashes and chaos," Quant. Finan. 2, 264-281.

Galam, S., Gefen, Y. \& Shapir, Y. [1982] "Sociophysics: A new approach of sociological collective behaviour. I. Mean-behaviour description of a strike," J. Math. Sociol. 9, 1-13.

Galam, S. \& Moscovici, S. [1991] "Towards a theory of collective phenomena: Consensus and attitude changes in groups," Eur. J. Soc. Psychol. 21, 49-74.

Gordon, M. B., Nadal, J.-P., Phan, D. \& Semeshenko, V. [2009] "Discrete choices under social influence: Generic properties," Math. Models Meth. Appl. Sci. 19, 1441-1481.

Granovetter, M. \& Soong, R. [1983] "Threshold models of diffusion and collective behavior," J. Math. Sociol. 9, 165-179.

Guckenheimer, J. \& Holmes, P. [1983] Nonlinear Oscillations, Dynamical Systems, and Bifurcations of Vector Fields (Springer-Verlag, NY, USA).
Harras, G., Tessone, C. J. \& Sornette, D. [2012] "Noiseinduced volatility of collective dynamics," Phys. Rev. E 85, 011150.

Johansen, A., Sornette, D. \& Ledoit, O. [1999] "Predicting financial crashes using discrete scale invariance," J. Risk 1, 5-32.

Johansen, A., Ledoit, O. \& Sornette, D. [2000] "Crashes as critical points," Int. J. Theor. Appl. Finan. 3, 219-255.

Kaizoji, T., Leiss, M., Saichev, A. \& Sornette, D. [2015] "Super-exponential endogenous bubbles in an equilibrium model of fundamentalist and chartist traders," J. Econ. Behav. Organ. 112, 289-310.

Kuznetsov, Y. [1998] Elements of Applied Bifurcation Theory (Springer, NY, USA).

Lempérière, Y., Deremble, C., Seager, P., Potters, M. \& Bouchaud, J. P. [2014] "Two centuries of trend following," J. Invest. Strateg. 3, 41-61.

Mandel, P. \& Erneux, T. [1984] "Laser Lorenz equations with a time-dependent parameter," Phys. Rev. Lett. 53, 1818-1820.

McCoy, B. M. \& Wu, T. T. [1973] The Two-Dimensional Ising Model (Harvard University Press, USA).

Montroll, E. W. \& Badger, W. W. [1974] Introduction to Quantitative Aspects of Social Phenomena (Gordon and Breach, NY, USA).

Nadal, J.-P., Phan, D., Gordon, M. B. \& Vannimenus, J. [2005] "Multiple equilibria in a monopoly market with heterogeneous agents and externalities," Quant. Finan. 5, 557-568.

Ollikainen, M. [2016] "Multiple market regimes in an equilibrium model of fundamentalist and noise traders," Master's thesis, ETH Zürich, 2016.

Orléan, A. [1995] "Bayesian interactions and collective dynamics of opinion: Herd behavior and mimetic contagion," J. Econ. Behav. Organ. 28, $257-274$.

Phan, D., Gordon, M. B. \& Nadal, J.-P. [2004] "Social interactions in economic theory: An insight from statistical mechanics," Cognitive Economics - An Interdisciplinary Approach (Springer, Berlin, Heidelberg), pp. 335-354.

Roncalli, T. [2017] "Keep up the momentum," SSRN, http://dx.doi.org/10.2139/ssrn.3083921.

Ross, A., Moskowitz, T. J., Israel, R. \& Serban, L. [2017] "Implementing momentum: What have we learned?" SSRN, http://dx.doi.org/10.2139/ssrn.3081165.

Schelling, T. C. [1971] "Dynamic models of segregation," J. Math. Sociol. 1, 143-186.

Sornette, D. [2003] Why Stock Markets Crash (Princeton University, Princeton, USA).

Sornette, D. \& Zhou, W.-X. [2006] "Importance of positive feedbacks and overconfidence in a self-fulfilling Ising model of financial markets," Physica A $\mathbf{3 7 0}$, $704-726$. 
Sornette, D., Woodard, R., Yan, W. \& Zhou, W.X. [2013] "Clarifications to questions and criticisms on the Johansen-Ledoit-Sornette financial bubble model," Physica A 392, 4417-4428.

Sornette, D. [2014] "Physics and financial economics (1776-2014): Puzzles, Ising and agent-based models," Rep. Prog. Phys. 77, 062001.

Weidlich, W. [1971] "The statistical description of polarization phenomena in society," Br. J. Math. Stat. Psychol. 24, 251-266.

Weidlich, W. [1991] "Physics and social science - The approach of synergetics," Phys. Rep. 204, 1-163.
Weidlich, W. [2003] "Sociodynamics — A systematic approach to mathematical modelling in the social sciences," Chaos Solit. Fract. 18, 431-437.

Weidlich, W. \& Huebner, H. [2008] "Dynamics of political opinion formation including catastrophe theory," J. Econ. Behav. Organ. 67, 1-26.

Zhou, W.-X. \& Sornette, D. [2007] "Self-fulfilling Ising model of financial markets," Eur. Phys. J. B 55, $175-181$. 